\title{
Circular RNA regulates the onset and progression of cancer through the mitogen-activated protein kinase signaling pathway (Review)
}

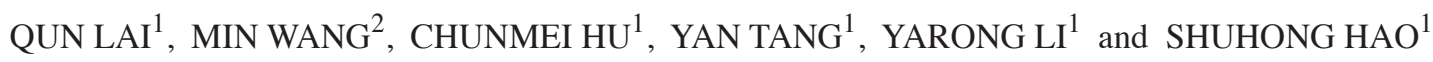 \\ Departments of ${ }^{1}$ Hematology and Oncology, and ${ }^{2}$ General Surgery, \\ The Second Hospital of Jilin University, Changchun, Jilin 130041, P.R. China
}

Received June 23, 2021; Accepted August 27, 2021

DOI: $10.3892 / \mathrm{ol} .2021 .13078$

\begin{abstract}
The rapid increase in cancer morbidity and mortality worldwide is a major challenge for public health providers. Therefore, there is an urgent need to explore the molecular mechanism of tumorigenesis and identify potential diagnostic biomarkers and therapeutic methods. Circular RNA (circRNA) is characterized by a stable structure and tissue-specific expression; these features are useful in medical research and clinical applications. In recent years, with the development of high-throughput sequencing technology, the potential use of circRNA in cancer prognosis and treatment has been extensively explored. Abnormal circRNA expression interferes with specific signaling pathways such as the MAPK pathway; this phenomenon may provide potential diagnostic biomarkers and new therapeutic targets. The present article discusses the research progress on the regulatory roles of MAPK/ERK pathway-related circRNA molecules in the development and progression of different types of tumors. This review may provide insight into the development of circRNA-based cancer management strategies.
\end{abstract}

\section{Contents}

1. Introduction

2. MAPK/ERK signaling pathway-related circRNA molecules and lung cancer

3. MAPK/ERK signaling pathway-related circRNA molecules and digestive system tumors

4. MAPK/ERK signaling pathway-related circRNA molecules and breast cancer

Correspondence to: Professor Shuhong Hao, Department of Hematology and Oncology, The Second Hospital of Jilin University, 265 Ziqiang Street, Changchun, Jilin 130041, P.R. China

E-mail:267311158@qq.com

Key words: non-coding RNA, circular RNA, MAPK signaling, cancer, tumor drug resistance
5. MAPK/ERK signaling pathway-related circRNA molecules and cervical cancer

6. MAPK/ERK signaling pathway-related circRNA molecules and urinary neoplasm

7. MAPK/ERK signaling pathway-related circRNA molecules and other malignancies

8. MAPK/ERK signaling pathway-related circRNA molecules and tumor drug resistance

9. Conclusions

\section{Introduction}

Non-coding RNA (ncRNA) is a type of functional RNA molecule that does not encode proteins, which can be classified into two categories: i) Housekeeper ncRNA, which includes ribosomal RNA, transfer RNA, small nuclear RNA and small nucleolar RNA; and ii) regulatory ncRNA, which includes microRNA (miRNA), long ncRNA and circular RNA (circRNA) $(1,2)$. circRNA is a group of endogenous ncRNA first discovered in RNA viruses by Sanger in the 1970s (3). circRNA is a single-stranded RNA molecule, often highly expressed in the cytoplasm of eukaryotic cells, and bound at the $3^{\prime}$ and 5 'ends to form a covalently closed loop structure, making them more stable and highly resistant to RNA degradation $(4,5)$. A study has shown that circRNA originates from both exon and intron regions and can pass through single exons, multiple exons and introns (exon-intron circRNA) or single intron sequences (circularized intron RNA) (6).

When initially discovered, circRNA molecules were regarded as post-transcriptional errors (7). However, with the emergence of various biomedical technologies, the biogenesis and function of circRNA have now been extensively explored. A previous study confirmed that circRNA can exhibit tissue- and developmental stage-specific expression and play important roles in multiple aspects of biology and disease (8). Several studies have shown that circRNA is involved in the occurrence and development of neurological (9) and cardiovascular diseases (10), as well as aging (11) and insulin secretion (12). In addition, circRNA may serve as a diagnostic or prognostic marker for a variety of cancer types, such as lung cancer (13), stomach cancer (14), colorectal cancer (CRC) (15) and 
hepatocellular carcinoma (HCC) (16). circRNA harbors single or multiple miRNA-binding sites, known as miRNA response elements, which competitively bind to specific miRNA targets and thus act as miRNA sponges, thereby reducing their expression and inhibiting their function. This leads to enhanced expression of the target genes $(17,18)$. For example, circular coiled-coil domain containing 66 (circ-CCDC66) promotes cell proliferation, invasion and metastasis in CRC by sponging miR-33b and miR-93 (19). Additionally, circRNA regulates the apoptosis, proliferation, migration, invasion and angiogenesis of cancer cells by regulating the expression of genes involved in cancer-related signaling pathways and epithelial-mesenchymal transformation (20-22). For instance, circular zinc finger with KRAB and SCAN domains 1 (circ-ZKSCAN1) promotes the proliferation of non-small cell lung cancer (NSCLC) cells by targeting miR-330-5p to inactivate MAPK signaling (23). Therefore, circRNA molecules may be used in clinical applications, as they are biologically important in oncogenesis and show potential as diagnostic or prognostic biomarkers and therapeutic targets.

The MAPK signaling pathway plays an important role in the survival, migration and drug resistance of human cancer cells; it also regulates the occurrence and development of various cancer types (24). The MAPK signaling pathway family consists of four main pathways: i) The ERK pathway; ii) the JNK pathway, iii) the p38/MAPK pathway; and iv) the ERK5/mitogen-activated protein kinase pathway (big MAP kinase 1) (Fig. 1) (25). The ERK-mediated MAPK signaling pathway is considered a classical MAPK signal transduction pathway (21). Several MAPK signaling pathways have been implicated in signal transduction from cell surface receptors, such as receptor tyrosine kinases (RTKs) or G-protein-coupled receptors, to the nucleus, leading to the activation of various cellular processes (26). Following ligand binding to the extracellular domain of the receptor, RTKs dimerize and promote kinase activity in the cytoplasmic domain (27). However, MAPK/ERK signal can be upregulated due to overexpression or abnormal activation of RTK or its direct downstream target RAS gene (28). RAS is the first intracellular effector in the ERK1/2 pathway; its activation is triggered by various extracellular stimuli, including growth factor-mediated activation of RTKs (24). The typical MAPK/ERK signaling pathway comprises A-RAF, B-RAF, RAF-1 or C-Raf kinases. After RAS activation, RAF activates MEK1 and MEK2 as a MAPK kinase kinase (MAPKKK), then promotes the activation of the ERK1 and ERK2 kinases, with ERK1 and ERK2 also involved in the final effects of the MAPK/ERK pathway (29). Once activated, ERK1/2 interacts with several nuclear and cytoplasmic effector genes, including activator protein-1, and plays a key role in regulating cell survival and proliferation $(29,30)$. Reactive oxygen species (ROS) can also regulate the MAPK/JNK signaling pathway. Oxidative stress activates apoptosis signal-regulated kinase 1, the upstream kinase of MAPK, and regulates the JNK pathway. Apoptosis is induced by the phosphorylation of MAPK kinase 4 (MKK4), MKK3 and MKK6 (31). The direct inhibition of MAPK phosphatase by ROS can also activate the MAPK/JNK pathway. Indeed, nicotinamide-adenine dinucleotide phosphate (NADPH) oxidase or ROS produced in mitochondria have been shown to inhibit the phosphatase and PI3K pathways inactivated by $\mathrm{JNK}$, resulting in the continuous activation of MAPK/JNK and MAPK/PI3K signaling pathways, leading to cell apoptosis $(32,33)$. Continuous activation of the MAPK/ERK pathway can lead to abnormal cell proliferation and cell cycle, inhibition of cell apoptosis and differentiation, as well as increased cell motility, migration and invasion. However, activation of the MAPK/JNK or p38MAPK pathway can lead to mitochondrial damage and cancer cell death, which is associated with the occurrence, development and prognosis of various tumors $(34,35)$.

A previous study has indicated that circRNA plays a key role in regulating cancer progression and interact with the MAPK, PI3K/AKT and Wnt/ $\beta$-catenin signaling pathways (36). MAPK/ERK1/2 signaling can promote cell proliferation and differentiation and accelerate cancer cell metastasis, while MAPK/JNK and MAPK/p38 signaling can induce cell apoptosis and inhibit cancer cell metastasis $(34,35)$. The multiple regulatory roles of the MAPK signaling pathway on the growth and development of cancer cells indicate the importance of the MAPK signaling pathway in the occurrence and development of cancer, which is worthy of further study (31-33). Hu et al (37) reported that circular ArfGAP with SH3 domain, ankyrin repeat and PH domain 1 (circ-ASAP1) promoted the proliferation and invasion of HCC cells by regulating the miR-326/miR-532-5p/MAPK1 axis and mediated tumor-associated macrophage infiltration by regulating miR-326/miR-532-5p/colony-stimulating factor-1 signaling. These studies provided insight into the metastasis of cancer and improved the understanding of the mechanisms of cancer development. The recent studies on MAPK/ERK signaling pathway-related circRNA molecules in specific cancers are summarized in Table I, and their regulatory mechanisms are shown in Fig. 2.

\section{MAPK/ERK signaling pathway-related circRNA molecules and lung cancer}

Lung cancer is one of the most common malignancies in humans and the leading cause of cancer-related death worldwide (38). Although immense progress has been made in the treatment of lung cancer, including in surgery, radiotherapy, chemotherapy and immunotherapy, the 5-year survival rate of patients with lung cancer remains only $15-20 \%$ due to the lack of early diagnosis and high frequency of metastasis and recurrence (39). Therefore, it is important to identify the signaling molecules involved in the abnormal changes that lead to the occurrence and development of lung cancer, in order to identify early biomarkers and reliable and effective therapeutic targets and to improve the prognosis of lung cancer.

Previous studies have suggested that circRNA acts as a miRNA sponge and plays a regulatory role in lung cancer $(40,41)$. One particular study demonstrated that the expression of circ-ZKSCAN1 was higher in NSCLC than in adjacent normal lung tissue (23). Moreover, Kaplan-Meier analysis revealed that the overall survival (OS) of patients with NSCLC and high circ-ZKSCAN1 expression was significantly shorter than that of patients with low circ-ZKSCAN1 expression. Consistent with these results, lung cancer cell proliferation was significantly reduced following circ-ZKSCAN1 knockout. These data indicate that circ-ZKSCAN1 is associated with the 


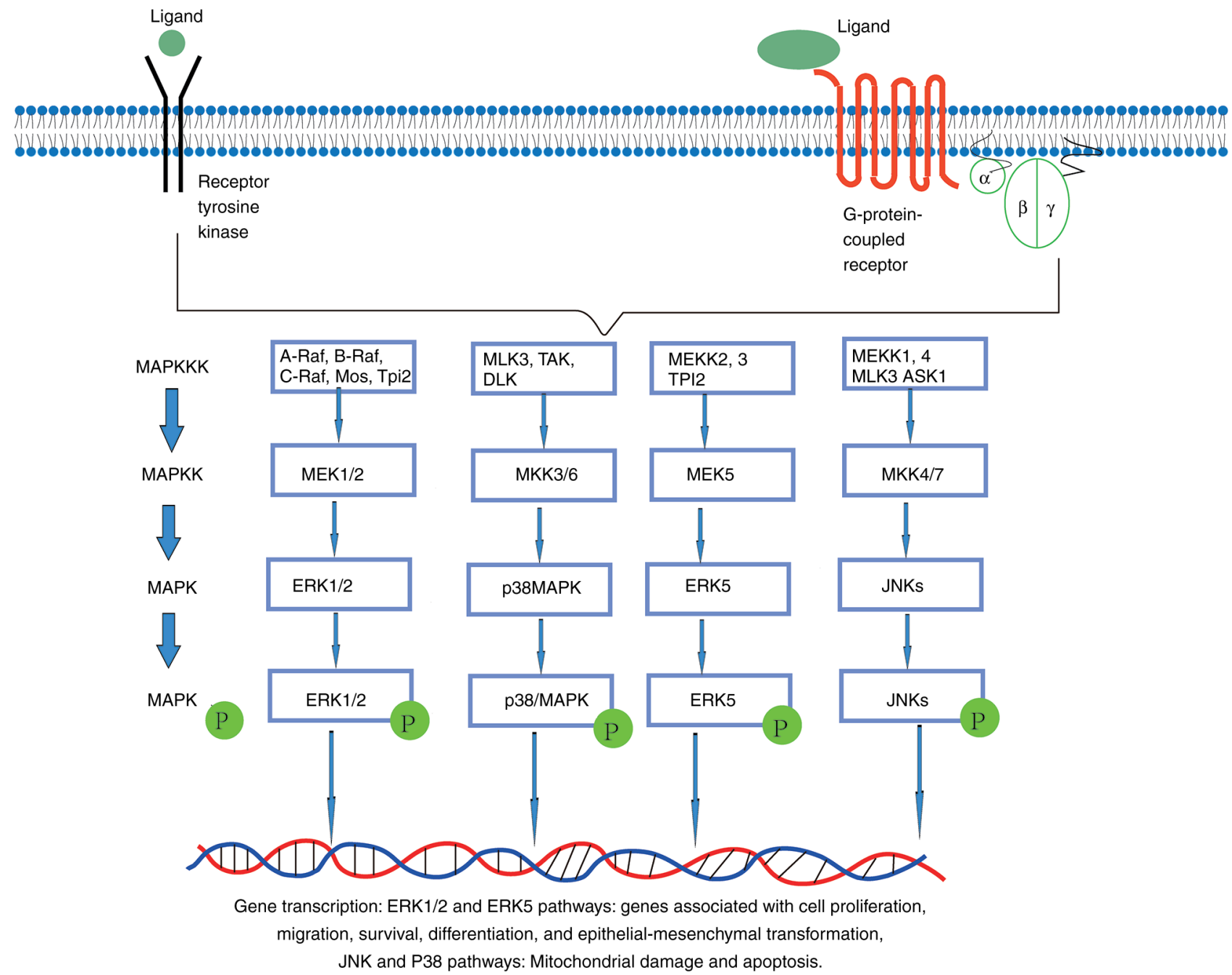

Figure 1. MAPK signaling pathways. ERK1/2, ERK5, JNKs and p38 proteins are classical components of MAPK signaling pathways according the traditional linear four-tiered organization. These pathways have been implicated in signal transduction from cell surface receptors (such as receptor tyrosine kinases or G-protein-coupled receptors) to the nucleus, leading to the activation of various cellular processes, including cell proliferation, survival, migration and differentiation. p-, phosphorylated.

occurrence, development and prognosis of NSCLC. Moreover, circ-ZKSCAN1 can sponge the oncogenic miR-330-5p to increase the expression of family with sequence similarity 83 member A, which inhibits the MAPK signaling pathway, significantly reducing the expression of effectors JNK, P38 and ERK (23). According to another study (42), the 3'-untranslated region of galectin-1 (Gal-1) contains a binding site for miR-22-3p, and miR-22-3p binds to circular fibroblast growth factor receptor (FGFR) 3 (circ-FGFR3) in NSCLC cells as a competitive endogenous RNA. Thus, circ-FGFR 3 acts as a sponge for miR-22-3p and upregulates Gal-1 mRNA expression, thereby activating the ERK1/2 signaling pathway, promoting tumor angiogenesis and inducing cancer cell invasion and proliferation (42). Zhang et al (43) also observed abnormally elevated circ 0001313 and reduced miR-452 levels in NSCLC cells. Moreover, circ0001313 silencing or miR-452 overexpression significantly reduced NSCLC cell proliferation and invasion. In addition, circ 0001313 could competitively bind to miR-452 to upregulate high mobility group box 3, which increased ERK1/2 and p38MAPK phosphorylation, thus activating the ERK/MAPK pathway and contributing to NSCLC development (43). Based on these studies, it may be hypothesized that MAPK/ERK signaling pathway-related circRNA molecules could be used as biomarkers and therapeutic targets for lung cancer. In the future, the design of drugs targeting the signaling molecules or kinases associated with circRNA or the MAPK signaling pathway may become possible.

\section{MAPK/ERK signaling pathway-related circRNA molecules and digestive system tumors}

Tumors of the digestive system are among the most common malignancies worldwide (44). Indeed, HCC, stomach cancer and CRC are the leading causes of digestive system cancer-related deaths, placing a heavy burden on societies worldwide (45). HCC alone is the fifth most common cancer worldwide. Several factors contribute to HCC development and progression (46). Uncontrolled cell proliferation associated with activation of the MAPK pathway has been defined as a major mechanism in HCC (47). MAPK14, a p38/MAPK, is a well-known serine/threonine kinase. Activation of $\mathrm{p} 38 / \mathrm{MAPK}$ signaling induces apoptosis in 


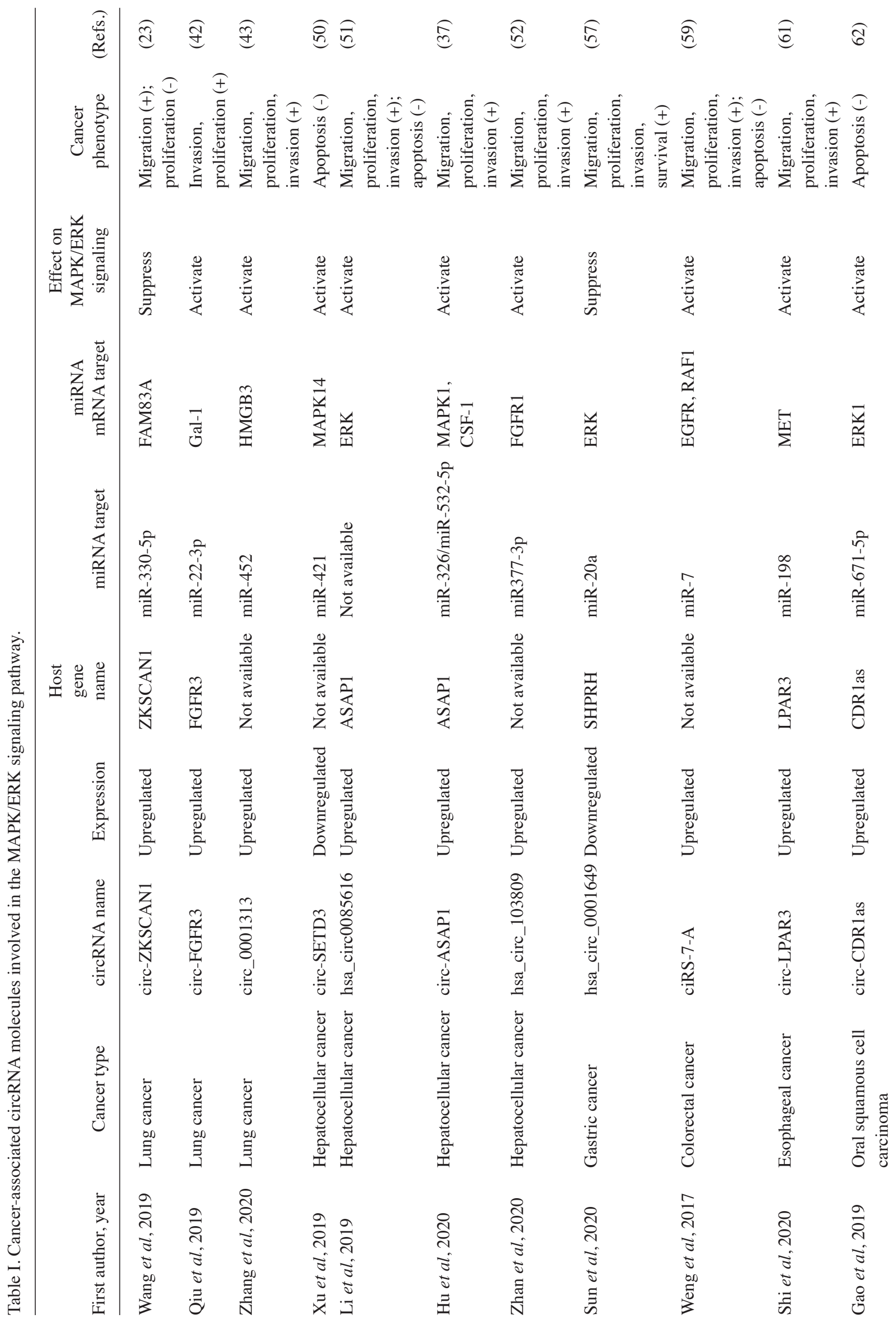




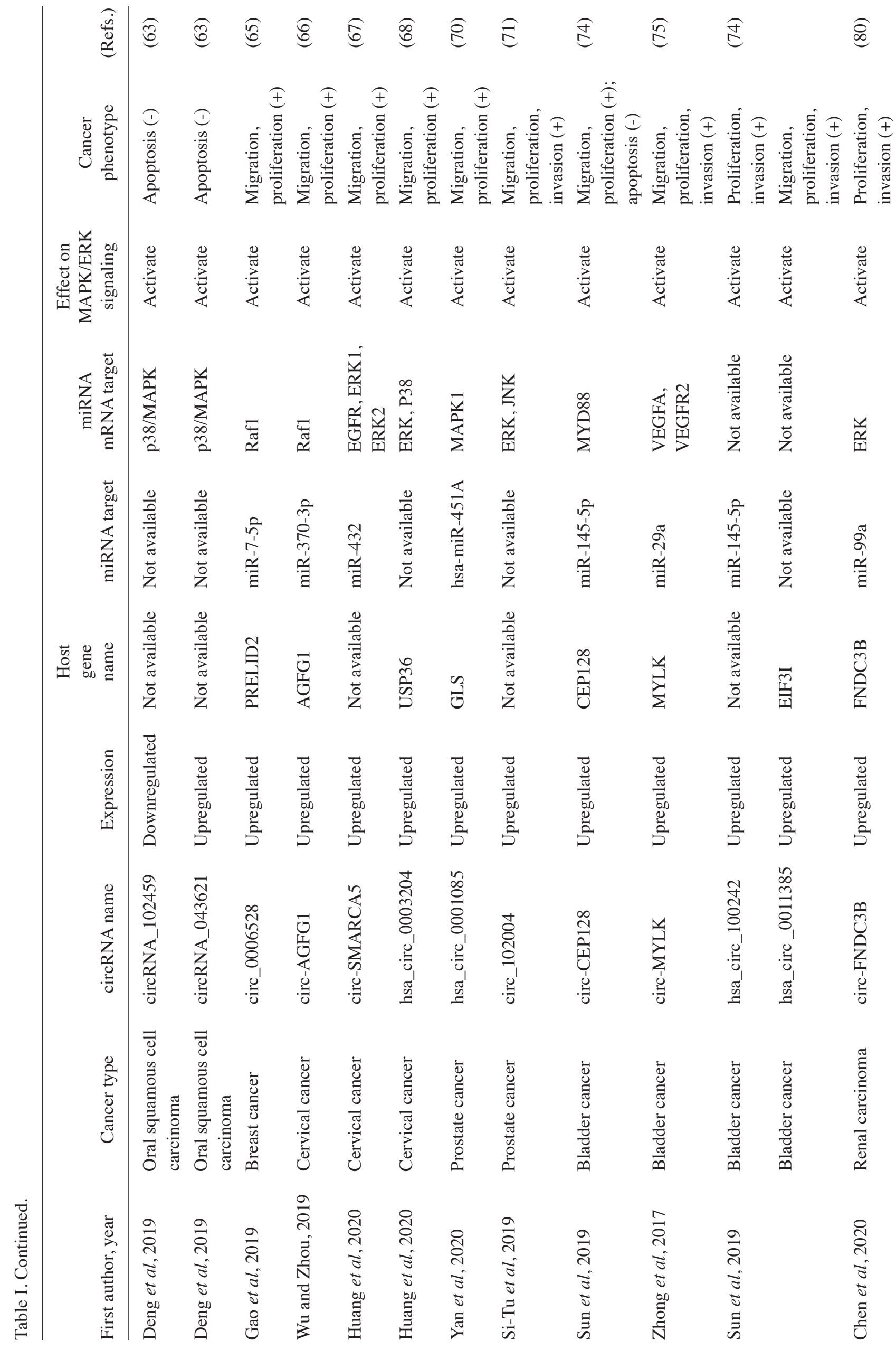


HCC cells via the mitochondrial apoptosis pathway of ROS $(48,49)$. In HCC, circular SET domain containing 3, actin histidine methyltransferase (circ-SETD3, also known as hsa_circ_0000567) is expressed at significantly lower expression in HCC than in paracancerous tissue samples. Moreover, the OS of patients with HCC and low circ-SETD3 expression is significantly shorter than that of patients with high circ-SETD3 expression (50). Thus, low expression of circ-SETD3 in HCC tissue predicts poor prognosis for patients with HCC. As a sponge for miR-421, circ-SETD3 upregulates the protein levels of p38/MAPKs and partially inhibits the growth and tumorigenesis of HCC cells (50). In addition, hsa_circ_0085616 is upregulated in HCC cells and promotes ERK phosphorylation levels; it also activates the MAPK pathway, thereby promoting the proliferation, migration and invasion of HCC cells in vitro (51). In another study, circ-ASAP1, a key regulator of HCC metastasis, was found to be highly expressed in the cytoplasm of in vivo HCC cells showing lung metastasis. circ-ASAP1 sponges miR-326 and miR-532-5p, increases the expression of MAPK1 (also known as ERK2), activates the ERK1/2 signaling pathway and promotes the proliferation of the HCC cells (37). Additionally, Zhan et al (52) suggested that the expression levels of hsa_circRNA_103809 was markedly higher in HCC samples than in tumor-adjacent samples and that patients with HCC and high hsa_circRNA_103809 expression showed a relatively lower survival rate than those with low hsa_circRNA_103809 expression. Additionally, the growth of HCC cells was inhibited after hsa_circRNA_103809 knockdown using small interfering RNA (siRNA). It was also shown that hsa_circRNA_103809 sponged miR-377-3p, which upregulated FGFR1 expression (52). FGFR1 is a member of the fibroblast growth receptor family that activates the RAS-RAF-MAPK (MEK)-ERK signaling pathway (53). Thus, the hsa circRNA_103809/miR-377-3p/FGFR1-MEK-ERK axis is a potential target for HCC treatment. hsa_circ_0001649 also plays an inhibitory role in various tumor types (54-56).

One study reported that the expression of hsa circ_0001649 in gastric cancer tissue is significantly lower than that in normal tissue. Flow cytometry analysis showed that if the expression of hsa_circ_0001649 was significantly upregulated in gastric cancer tissue, it could stimulate cell apoptosis and reduce the migration and invasion ability of gastric cancer cells. This is because hsa_circ_0001649 can sponge mir-20a and inhibit the ERK and Wnt/ $\beta$-catenin signaling pathways, as well as cell growth and development, leading to apoptosis (57). The roles of additional MAPK/ERK signaling pathway-associated circRNA molecules in gastric cancer requires further analysis, which may lead to the identification of potential biomarkers of tumorigenesis, targeted therapy and prognosis.

miR-7 can effectively reduce the expression levels of EGFR and RAF1, an important MAPK family member, and strongly inhibit the EGFR/RAF1/MAPK pathway (58). Weng et al (59) found that ciRS-7-A expression was significantly higher in CRC than in normal tissue. High ciRS-7-A expression positively correlated with CRC lymph node metastasis, and multivariate survival analysis revealed that ciRS-7-A was an independent risk factor for OS, suggesting its potential carcinogenic effect 


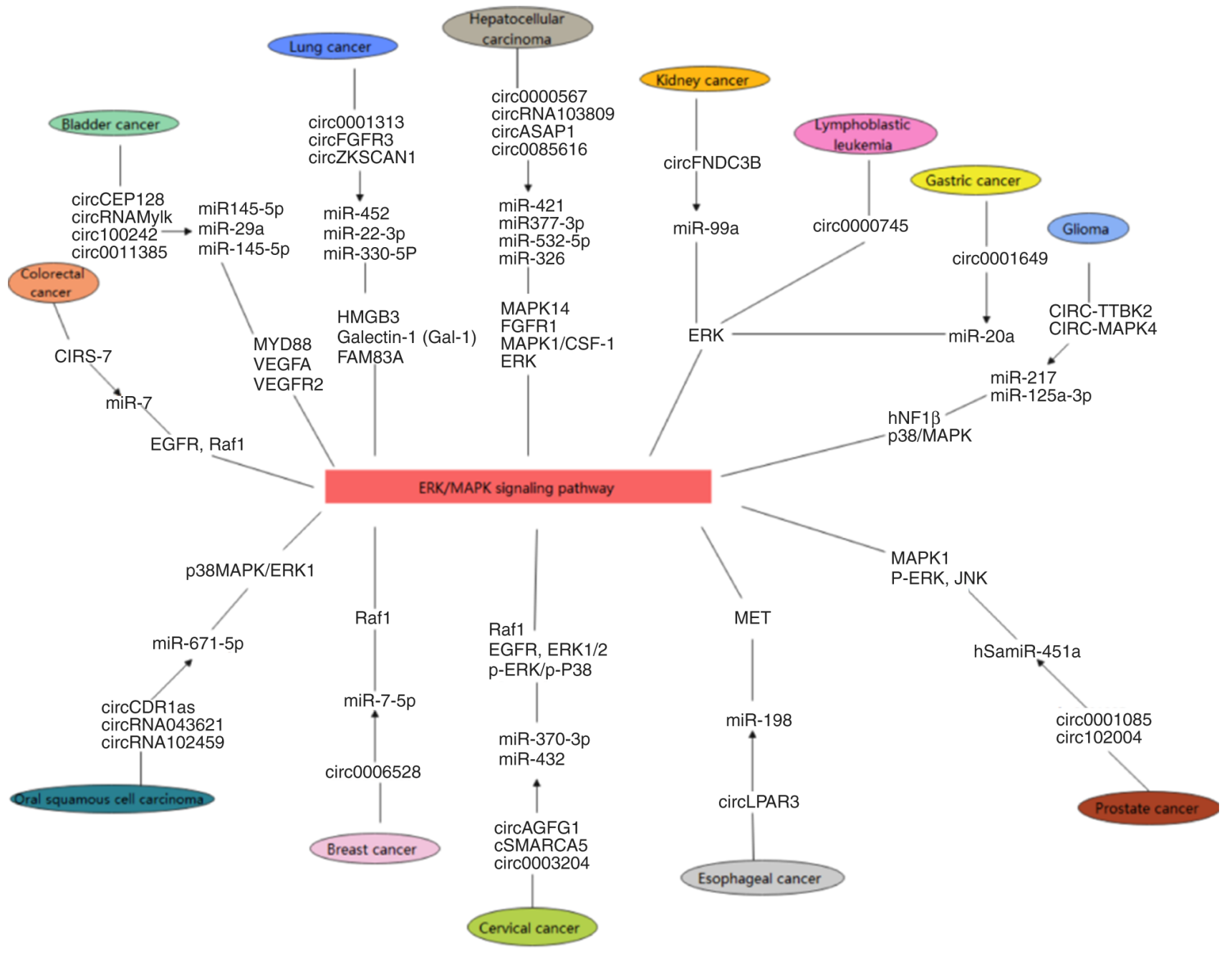

Figure 2. Human cancer-associated circRNA molecules and the MAPK/ERK signaling pathway. The MAPK/ERK signaling pathway plays a critical role in the progression of human cancer. circRNA molecules associated with MAPK/ERK signaling adsorb miR targets by sponging them, inhibiting their effects on target genes and regulating them. This, in turn, activates or inhibits MAPK/ERK signaling and regulates the growth, metastasis and apoptosis of cancer cells. circRNA, circular RNA; miR, microRNA.

in CRC and prognostic effect. ciRS-7-A upregulation in CRC induced EGFR/RAF1/MAPK signaling by sponging miR-7, thereby inhibiting the proliferation, migration, invasion and apoptosis of cancer cells (59).

Esophageal cancer (EC) is the sixth leading cause of cancer-related death worldwide, and its high mortality is associated with distant organ recurrence and metastasis $(60,61)$. Previous studies have suggested that circular lysophosphatidic acid receptor 3 (circ-LPAR3) plays an important role in EC occurrence and metastasis (61). Shi et al (61) demonstrated that circ-LPAR3 expression was markedly higher in esophageal squamous cell carcinoma (ESCC) than in paracarcinoma tissue samples. Similarly, its high expression promoted the migration and invasion of ESCC cells in a Transwell assay. circ-LPAR3 acted as a sponge for miR-198 in ESCC cells to regulate the phosphorylation of downstream MAPK and AKT signaling proteins, thereby promoting ESCC occurrence and progression (61).

It has been reported that circular CDR1 antisense (circ-CDR1as) expression is markedly higher in oral squamous cell carcinoma (OSCC) than in normal oral tissue, and Kaplan-Meier analysis demonstrated that patients with higher
circ-CDR 1as expression have a shorter postoperative survival time than those with lower circ-CDR1as expression (62). circ-CDRlas upregulation is associated with poor prognosis in patients with OSCC. The mechanism is that circ-CDR1as acts as a sponge for miR-671-5p in the hypoxic microenvironment to promote hypoxia-induced autophagy and further promote activation of AKT and ERK1 signaling and inhibit cancer cell apoptosis (62). Therefore, autophagy inhibitors combined with circ-CDR1as may represent new therapeutic options for the treatment of OSCC (62). According to another study (63), circRNA_102459 and circRNA_043621 are inhibitors and promoters of OSCC, respectively. circRNA_102459 overexpression or circRNA_043621 knockdown causes MAPK pathway inhibition, thus promoting OSCC cell apoptosis (63). However, whether circRNA_102459 and circRNA_043621 act as miRNA sponges requires further in-depth studies (63).

Although further studies of circRNA and gastrointestinal tumors are needed, these findings demonstrate that MAPK/ERK pathway-related circRNA molecules may be useful for diagnosis and prognosis in gastrointestinal tumors, as well as the development of new therapeutic strategies. 


\section{MAPK/ERK signaling pathway-related circRNA molecules and breast cancer}

Breast cancer $(\mathrm{BC})$ is the most common cause of cancer-related death in women worldwide (44). Despite improvement in surgery, as well as endocrine therapy, and targeted therapy in recent years, BC mortality remains high, due to late diagnosis and high metastasis and recurrence rates (64). Therefore, it is important to understand the molecular pathways involved in the pathogenesis of $\mathrm{BC}$. Through Using quantitative real-time PCR analysis, Gao et al (65) showed that circ_0006528 was significantly upregulated in BC compared with adjacent non-tumor tissue, which was significantly associated with advanced tumor-node-metastasis stage and poor prognosis. Moreover, Kaplan-Meier survival analysis indicated that patients with high circ_0006528 expression had poorer relapse-free survival and OS than those with low circ_0006528 expression. Additionally, siRNA-mediated circ_0006528 knockdown impaired the proliferation of BC cells. A study showed that RAF1 upregulation can significantly increase MEK1/2 and ERK1/2 phosphorylation, thus activating the MAPK/ERK signaling pathway, which promotes the growth, invasion and migration of BC.circ_0006528 sponges mir-7-5p, which attenuates the inhibitory effect of this miRNA on RAF1 and upregulates RAF1 in vivo (65). Thus, circ_0006528 can promote DNA synthesis, cell proliferation and BC invasion and migration (65). In conclusion, circ_0006528 may be involved in $\mathrm{BC}$ occurrence, making it a potential therapeutic target in patients with $\mathrm{BC}$. This may become an important direction of $\mathrm{BC}$ research in the future.

\section{MAPK/ERK signaling pathway-related circRNA molecules and cervical cancer}

Cervical cancer (CC) is a common malignant tumor and the second leading cause of cancer-related death in women (44). Various circRNA molecules have been reported to be upregulated in $\mathrm{CC}$ and are involved in the migration, invasion, angiogenesis and proliferation of CC cells through the MAPK/ERK signaling pathway. For example, Wu et al (66) found that circular ArfGAP with FG repeats 1 (circ-AGFG1) expression was higher in $\mathrm{CC}$ cells than in normal cells and that the viability and proliferation of $\mathrm{CC}$ cells was inhibited following circ-AGFG1 knockdown. circ-AGFG1 stimulates the RAF/MEK/ERK pathway by sponging miR-370-3p to increase the levels of RAF1, phosphorylated (p-)RAF1, p-MEK1/2 and p-ERK1/2 proteins (66). Huang et al (67) demonstrated that circular SWI/SNF related, matrix associated, actin dependent regulator of chromatin, subfamily a, member 5 (circ-SMARCA5) expression was significantly higher in $\mathrm{CC}$ than in non-tumor tissue samples and that circ-SMARCA5 silencing inhibited the proliferation and invasion of CC cells. Moreover, circ-SMARCA5 sponged miR-432 to inhibit EGFR downregulation, thereby activating the ERK1/2 signaling pathway and promoting CC progression (67). Additionally, hsa_circ_0003204 upregulation has been reported to promote the proliferation, migration and invasion of CC cells by activating the MAPK signaling pathway, although its miRNA target has not been confirmed (68).

\section{MAPK/ERK signaling pathway-related circRNA molecules and urinary neoplasms}

Prostate cancer (PCa) is the most common urinary system-associated malignancy in men (69). The incidence and mortality of PCa in China are increasing, and its metastasis and recurrence are essential reasons for its poor prognosis (69). It has been reported that hsa_circ_0001085 upregulates MAPK1 protein expression levels by sponging hsa-miR-451a, promotes MAPK signaling and PCa cell proliferation and metastasis and induces PCa cell epithelial-mesenchymal transformation through the MAPK pathway (70). Si-Tu et al (71) found that circ-102004 upregulation was significantly greater in $\mathrm{PCa}$ than in benign prostatic hyperplasia tissue. Moreover, both the migration and invasion of PCa cells increased following circ-102004 overexpression. circ-102004 overexpression in $\mathrm{PCa}$ leads to significant upregulation of p-ERK, p-Akt, p-JNK, JNK, $\beta$-catenin and GL11, which activates the MAPK pathway. Thus, circ-102004 plays an oncogenic role in $\mathrm{PCa}$ by affecting the MAPK signaling pathways known to promote cancer development and progression (71).

Bladder cancer represents the second most common neoplasm among urological malignancies and the fourth in general among men (72). Progress in the treatment of $\mathrm{BCa}$ remains limited, and this cancer is associated with a high mortality rate (73). It has been reported that circular centrosomal protein 128 (circ-CEP128) is highly expressed in BCa and that circ-CEP128 knockout can increase the apoptosis of BCa cells, induce cell cycle arrest and inhibit tumor growth. circ-CEP128 overexpression in $\mathrm{BCa}$ upregulates the expression of MyD88 and the phosphorylation of MAPK signaling pathway-related proteins (p38, ERK and JNK) by sponging miR-145-5p, activates the MAPK signaling pathway and further promotes the proliferation and motility of BCa cells (74). Another study showed that circular myosin light chain kinase, which sponges miR-29a, eliminated the inhibitory effect on target gene VEGFA, promoted the phosphorylation of VEGFR2, RAF-1, MEK1/2 and ERK1/2, induced epithelial-mesenchymal transformation, activated the RAS/ERK signaling pathway and participated in the progression of $\mathrm{BCa}$ (75). In addition, Lu et al (76) demonstrated that hsa_circ_0011385,circ_100242 and miR-145 affect the MAPK signaling pathway through a circRNA-miRNA-mRNA network and drive the proliferation of BCa cells; however, identifying their direct targets requires further analysis. These results suggest that circRNA networks and signaling pathways are potential clinical markers and therapeutic targets for patients with $\mathrm{BCa}$ (76).

Renal carcinoma ( $\mathrm{RC}$ ) is a common malignancy, and increasing evidence suggests that circRNA is involved in RC development and progression $(77,78)$. Circular fibronectin type III domain containing 3B (circ-FNDC3B), a circRNA spliced from FNDC3B, has been reported to promote tumor growth (79). Moreover, Chen et al (80) found that circ-FNDC3b expression was significantly increased in RC tissue samples and that circ-FNDC3b negatively regulated miR-99a and activated the MAPK/ERK signaling pathway, thus promoting the survival, clone formation and migration of RC cells.

Further studies on circRNA molecules related to the MAPK/ERK signaling pathway are needed to determine their roles and metastasis mechanism in the pathogenesis of urinary 
system-associated tumors and to identify potential therapeutic targets.

\section{MAPK/ERK signaling pathway-related circRNA molecules and other malignancies}

circRNA has been reported to regulate the occurrence and progression of acute lymphoblastic leukemia (81), multiple myeloma (MM) (82) and glioma (83) through the typical MAPK/ERK signaling pathway. It has been reported that circ-0000745 expression was significantly higher in human leukemia cell lines (Kasumi-1 and KG-1 cells) than in human primary $\mathrm{T}$ lymphocytes. Furthermore, flow cytometry analysis showed that the frequency of apoptotic cells was reduced after circ-0000745 overexpression. In addition, circ-0000745 overexpression induces leukemia cell proliferation by increasing ERK phosphorylation, thus activating the ERK signaling pathway and promoting the development of acute lymphoblastic leukemia (81).

$\mathrm{MM}$, the second most common hematologic malignancy, is a malignancy originating in the bone marrow characterized by uncontrolled growth of monoclonal plasma cells and the production of excessive monoclonal immunoglobulins (84). In recent years, remarkable advancements have been made in treatment strategies, such as stem cell transplantation and targeted therapy, prolonging the survival of patients with MM (85). Although chronic disease can be controlled, relapse and multidrug resistance remain major problems $(86,87)$. Zhou et al (82) demonstrated that 122 and 260 circRNA molecules were upregulated and downregulated in MM, respectively. Bioinformatics prediction of the functions of these circRNA candidates suggested that they were involved in tumor signaling pathways, such as MAPK and VEGF, by sponging miRNA targets, thereby affecting the pathogenesis of MM. However, the association between circRNA and the prognosis of MM requires further analysis.

Glioma is the most common and most lethal tumor of the primary nervous system in adults (88). Zheng et al (83) reported that the expression of circular $\tau$ tubulin kinase 2 (circ-TTBK2) was significantly higher in glioma tissue compared with that in normal brain tissue, as well as cells. In addition, circ-TTBK2 overexpression upregulated the expression of its target gene, HNF1 homeobox B, by sponging miR-217, thereby activating derlin-1 and the PI3K/AKT and ERK pathways. He et al (89) showed that circ-MAPK4 was upregulated in glioma cell lines compared with that in glial cell lines, and inhibition of circ-MAPK4 resulted in a significant decrease in the number of glioma cells, demonstrating that circ-MAPK4 markedly enhanced the survival of glioma cells. The possible mechanism is that circ-MAPK4 regulates the development of glioma by regulating the phosphorylation of p38/MAPKK (89).

\section{MAPK/ERK signaling pathway-related circRNA molecules and tumor drug resistance}

At present, chemotherapy resistance and relapse remain major challenges in cancer treatment. circRNA-induced dysregulation of the MAPK pathway has been observed to cause therapeutic resistance in lung adenocarcinoma, with increased circRNA CCDC66 levels leading to EGFR overexpression (90). EGFR is an important growth factor, and its overexpression promotes resistance to radiation and chemotherapy by activating MAP2K and MAP3K of the MAPK pathway; the MEK/ERK cascade was shown to be exclusively deactivated when EGFR expression is inhibited $(91,92)$. In this regard, EGFR inhibitors or RAS/MAPK axis inhibitors can be considered for future clinical use to overcome drug resistance. However, crosstalk between the RAS/MAPK pathway and other pathways may cause primary or acquired resistance to treatment with RAS/MAPK axis inhibitors. Nevertheless, combination therapy leading to inactivation of the RAS/MAPK pathway and PI3K/Akt pathway may overcome the escape mechanism (93). In acute myeloid leukemia, the upregulation of circRNA in drug-resistant cells is mainly related to the MAPK and mTOR/Akt signaling pathways, and blocking the PI3K/Akt signaling pathway can lead to downregulation of P-glycoprotein and multidrug resistance associated protein 1 expression and restore sensitivity to chemotherapeutic drugs (94). Although the specific mechanism of action has not been clarified, these results indicate new approaches for overcoming resistance to therapy in acute myeloid leukemia.

\section{Conclusions}

Cancer and cancer-related mortality rates have been increasing worldwide and are expected to become the leading cause of death and the most significant obstacle to increasing life expectancy in the 21st century (44). With new advances in precision medicine, great progress has been made in early detection technology, radiotherapy and chemotherapy, targeted therapy and immunotherapy; however, frequent recurrence, metastasis and resistance to chemotherapy lead to poor prognosis and remain a major obstacle to cancer treatment. Therefore, the mechanism of cancer occurrence and development should be further studied to identify more accurate and effective diagnostic and prognostic biomarkers, as well as potential targets for cancer treatment.

The discovery of circRNA in the human genome has changed the way cancer research is conducted,and it has been shown that with the application of high-throughput sequencing, circRNA molecules are easier to detect than miRNA (95). circRNA exerts unique functions in different tissue types and developmental stages and are highly expressed in different cancer cell lines (86). In fact, circRNA has other functions in addition to acting as miRNA sponge. For example, circRNA molecules can act as regulators of transcription and splicing (96) or adaptors for protein-protein interactions (97) and may be involved in ribosomal RNA processing (98). Thus, the sponge function of circRNA is the most widely and clearly studied in cancer research. However, other functions remain important, and a comprehensive understanding of these functions is needed to accurately understand the interactions between miRNA, circRNA and their target genes, how they coordinate to promote the development of human diseases and how circRNA expression or degradation is regulated in specific disease environments. All these require more systematic and genome-wide investigation and analysis. In conclusion, the discovery of more accurate cancer therapeutic targets and drug therapies to which cancers are less resistant will further contribute to the development of circRNA-based cancer therapy. 
The MAPK/ERK pathway plays a central role in human cancer and is activated in various tumor types. Several of its components have been identified as oncogenes, leading to increased interest in this pathway as a potential cancer treatment option (99). circRNA affects biological processes by activating or inhibiting the MAPK/ERK pathway. In lung cancer, HCC, $\mathrm{CC}$ and other cancer types, most circRNA molecules are upregulated and bind miRNA targets to abrogate their inhibitory effect on target genes. This promotes the expression levels of the target genes, thus activating the MAPK/ERK pathway and promoting proliferation, migration, invasion, whilst inhibiting in cancer cells. The aforementioned study suggests that the association between circRNA and the MAPK/ERK pathway can be targeted for cancer diagnosis and are prognostic biomarkers for cancer therapy. However, cell proliferation is a complex process. Besides interacting with the MAPK pathway, circRNA interacts with other signaling pathways, such as the PI3K/AKT intracellular signaling pathway (100) and the Wnt/ $\beta$-catenin pathway $(101,102)$; the mechanisms of interaction between these pathways are important and complex. In addition, the MAPK/ERK pathway typically involves crosstalk or interdependent signals with other signaling pathways, which jointly influence the occurrence and development of cancer. Crosstalk between the MAPK/ERK pathway and parallel pathways such as the PI3K/AKT pathway is also key to abnormal proliferation (27). Therefore, studies of the effect of circRNA and MAPK/ERK pathways on tumors and cross-effects of other pathways should be considered.

In summary, circRNA molecules associated with the MAPK/ERK signaling pathway play a key role in the occurrence, development, diagnosis, treatment and prognosis of various cancer types. circRNA promotes cancer development by binding to miRNA as a sponge to eliminate the inhibition of target genes and regulate key proteins in the MAPK/ERK signaling pathway. circRNA and the MAPK pathway are typically interrelated and cooperate to play a role in cell function. Therefore, circRNA or cellular signaling pathways alone may not be sufficient to drive cellular signaling to influence cancer development. The future research direction is to predict and diagnose cancer by detecting circRNA levels in relevant tissue types in vivo. It is necessary to further study the role of circRNA in different signaling pathways in relation to the occurrence and development of cancer. This may provide insight into the mechanism of drug resistance and contribute to the development of potential targets for cancer diagnosis, prognosis and treatment.

\section{Acknowledgements}

Not applicable.

\section{Funding}

This work was supported by The Science and Technology Department of Jilin Province (grant nos. 20190201050JC and 20200201543JC).

\section{Availability of data and materials}

Not applicable.

\section{Authors' contributions}

QL and SH were responsible for the conception or design of the work and were major contributors to the writing of the manuscript. $\mathrm{CH}$ and YT participated in the coordination of the study and reviewed the manuscript. YL was responsible for the revision of the manuscript, MW was responsible for conception and design of the work. All authors have read and approved the final manuscript.

\section{Ethics approval and consent to participate}

Not applicable.

\section{Patient consent for publication}

Not applicable.

\section{Competing interests}

The authors declare that they have no competing interests.

\section{References}

1. Hsiao KY, Sun HS and Tsai SJ: Circular RNA-new member of noncoding RNA with novel functions. Exp Biol Med (Maywood) 242: 1136-1141, 2017.

2. Lei B, Tian Z, Fan W and Ni B: Circular RNA: A novel biomarker and therapeutic target for human cancers. Int J Med Sci 16: 292-301, 2017.

3. Sanger HL, Klotz G, Riesner D, Gross HJ and Kleinschmidt AK: Viroids are single-stranded covalently closed circular RNA molecules existing as highly base-paired rod-like structures. Proc Natl Acad Sci USA 73: 3852-3856, 1976.

4. Salzman J, Chen RE, Olsen MN, Wang PL and Brown PO: Cell-type specific features of circular RNA expression. PLoS Genet 9: e1003777, 2013.

5. Afonina ZA, Myasnikov AG, Shirokov VA, Klaholz BP and Spirin AS: Formation of circular polyribosomes on eukaryotic mRNA without cap-structure and poly(A)-tail: A cryo electron tomography study. Nucleic Acids Res 42: 9461-9469, 2014.

6. Shen T, Han M, Wei G and Ni T: An intriguing RNA species-perspectives of circularized RNA. Protein Cell 6: 871-880, 2015.

7. Wang Y, Mo Y, Gong Z, Yang X, Yang M, Zhang S, Xiong F, Xiang B, Zhou M, Liao Q, et al: Circular RNAs in human cancer. Mol Cancer 16: 25, 2017.

8. Liu J, Liu T, Wang X and He A: Circles reshaping the RNA world: From waste to treasure. Mol Cancer 16: 58, 2017.

9. Bramham CR and Wells DG: Dendritic mRNA: Transport, translation and function. Nat Rev Neurosci 8: 776-789, 2007.

10. Burd CE, Jeck WR, Liu Y, Sanoff HK, Wang Z and Sharpless NE: Expression of linear and novel circular forms of an INK4/ARF-associated non-coding RNA correlates with atherosclerosis risk. PLoS Genet 6: e1001233, 2010.

11. Abdelmohsen K, Panda AC, De S, Grammatikakis I, Kim J, Ding J, Noh JH, Kim KM, Mattison JA, de Cabo R and Gorospe M: Circular RNAs in monkey muscle: Age-dependent changes. Aging (Albany NY) 7: 903-910, 2015.

12. $\mathrm{Xu} \mathrm{H}$, Guo S, Li W and Yu P: The circular RNA Cdrlas, via miR-7 and its targets, regulates insulin transcription and secretion in islet cells. Sci Rep 5: 12453, 2015.

13. de Fraipont F, Gazzeri S, Cho WC and Eymin B: Circular RNAs and RNA splice variants as biomarkers for prognosis and therapeutic response in the liquid biopsies of lung cancer patients. Front Genet 10: 390, 2019.

14. Rao M, Zhu Y, Qi L, Hu F and Gao P: Circular RNA profiling in plasma exosomes from patients with gastric cancer. Oncol Lett 20: 2199-2208, 2020.

15. Shen T, Cheng X, Liu X, Xia C, Zhang H, Pan D, Zhang X and Li Y: Circ_0026344 restrains metastasis of human colorectal cancer cells via miR-183. Artif Cells Nanomed Biotechnol 47: 4038-4045, 2019. 
16. Qiu L, Wang T, Ge Q, Xu H, Wu Y, Tang Q and Chen K: Circular RNA signature in hepatocellular carcinoma. J Cancer 10 : 3361-3372, 2019

17. Kristensen LS, Hansen TB, Ven $\varnothing$ MT and Kjems J: Circular RNAs in cancer: Opportunities and challenges in the field. Oncogene 37: 555-565, 2018.

18. Memczak S, Jens M, Elefsinioti A, Torti F, Krueger J, Rybak A Maier L, Mackowiak SD, Gregersen LH, Munschauer M, et al: Circular RNAs are a large class of animal RNAs with regulatory potency. Nature 495: 333-338, 2013.

19. Hsiao KY, Lin YC, Gupta SK, Chang N, Yen L, Sun HS and Tsai SJ: Noncoding effects of circular RNA CCDC66 promote colon cancer growth and metastasis. Cancer Res 77: 2339-2350, 2017.

20. Qu S, Liu Z, Yang X, Zhou J, Yu H, Zhang R and Li H: The emerging functions and roles of circular RNAs in cancer. Cancer Lett 414: 301-309, 2018.

21. Wang L, Tong X, Zhou Z, Wang S, Lei Z, Zhang T, Liu Z, Zeng Y, Li C, Zhao J, et al: Circular RNA hsa_circ_0008305 (circPTK2) inhibits TGF- $\beta$-induced epithelial-mesenchymal transition and metastasis by controlling TIF $1 \gamma$ in non-small cell lung cancer. Mol Cancer 17: 140, 2018.

22. Li Y, Wan B, Liu L, Zhou L and Zeng Q: Circular RNA circMTO1 suppresses bladder cancer metastasis by sponging miR-221 and inhibiting epithelial-to-mesenchymal transition. Biochem Biophys Res Commun 508: 991-996, 2019.

23. Wang Y, Xu R, Zhang D, Lu T, Yu W, Wo Y, Liu A, Sui T, Cui J, Qin Y, et al: Circ-ZKSCAN1 regulates FAM83A expression and inactivates MAPK signaling by targeting miR-330-5p to promote non-small cell lung cancer progression. Transl Lung Cancer Res 8: 862-875, 2019.

24. De Luca A, Maiello MR, D'Alessio A, Pergameno M and Normanno N: The RAS/RAF/MEK/ERK and the PI3K/AKT signalling pathways: Role in cancer pathogenesis and implications for therapeutic approaches. Expert Opin Ther Targets 16 (Suppl 2): S17-S27, 2012.

25. Chen T, Xiao Q, Wang X, Wang Z, Hu J, Zhang Z, Gong Z and Chen $S$ : miR-16 regulates proliferation and invasion of lung cancer cells via the ERK/MAPK signaling pathway by targeted inhibition of MAPK kinase 1 (MEK1). J Int Med Res 47: 5194-5204, 2019.

26. Delire B and Stärkel P: The Ras/MAPK pathway and hepatocarcinoma: Pathogenesis and therapeutic implications. Eur J Clin Invest 45: 609-623, 2015.

27. Shaw RJ and Cantley LC: Ras, PI(3)K and mTOR signalling controls tumour cell growth. Nature 441: 424-430, 2006.

28. Burotto M, Chiou VL, Lee JM and Kohn EC: The MAPK pathway across different malignancies: A new perspective. Cancer 120: 3446-3456, 2014.

29. Santarpia L, Lippman SM and El-Naggar AK: Targeting the MAPK-RAS-RAF signaling pathway in cancer therapy. Expert Opin Ther Targets 16: 103-119, 2012.

30. Fremin C and Meloche S: From basic research to clinical development of MEK1/2 inhibitors for cancer therapy. J Hematol Oncol 3: 8,2010.

31. Ichijo H, Nishida E, Irie K, ten Dijke P, Saitoh M, Moriguchi T, Takagi M, Matsumoto K, Miyazono K and Gotoh Y: Induction of apoptosis by ASK1, a mammalian MAPKKK that activates SAPK/JNK and p38 signaling pathways. Science 275: 90-94, 1997.

32. Liu RM, Choi J, Wu JH, Gaston Pravia KA, Lewis KM, Brand JD, Mochel NS, Krzywanski DM, Lambeth JD, Hagood JS, et al: Oxidative modification of nuclear mitogen-activated protein kinase phosphatase 1 is involved in transforming growth factor beta1-induced expression of plasminogen activator inhibitor 1 in fibroblasts. J Biol Chem 285: 16239-16247, 2010.

33. Robinson KA, Stewart CA, Pye QN, Nguyen X, Kenney L, Salzman S, Floyd RA and Hensley K: Redox-sensitive protein phosphatase activity regulates the phosphorylation state of p38 protein kinase in primary astrocyte culture. J Neurosci Res 55: 724-732, 1999

34. Maurer G, Tarkowski B and Baccarini M: Raf kinases in cancer-roles and therapeutic opportunities. Oncogene 30: 3477-3488, 2011

35. Ray PD, Huang BW and Tsuji Y: Reactive oxygen species (ROS) homeostasis and redox regulation in cellular signaling. Cell Signal 24: 981-990, 2012

36. Yang Z, Xie L, Han L, Qu X, Yang Y, Zhang Y, He Z, Wang Y and Li J: Circular RNAs: Regulators of cancer-related signaling pathways and potential diagnostic biomarkers for human cancers. Theranostics 7: 3106-3117, 2017
37. Hu ZQ, Zhou SL, Li J, Zhou ZJ, Wang PC, Xin HY, Mao L, Luo CB, Yu SY, Huang XW, et al: Circular RNA sequencing identifies CircASAP1 as a key regulator in hepatocellular carcinoma metastasis. Hepatology 72: 906-922, 2020.

38. Siegel RL, Miller KD and Jemal A: Cancer statistics, 2018 CA Cancer J. Clin 68: 7-30, 2018

39. Bagchi S: Lung cancer survival only increases by a small amount despite recent treatment advances. Lancet Respir Med 5: 169,2017

40. Jin X, Guan Y, Sheng H and Liu Y: Crosstalk in competing endogenous RNA network reveals the complex molecular mechanism underlying lung cancer. Oncotarget 8: 91270-91280, 2017.

41. Zhang C, Ma L, Niu Y, Wang Z, Xu X, Li Y and Yu Y: Circular RNA in lung cancer research: Biogenesis, functions, and roles. Int J Biol Sci 16: 803-814, 2020.

42. Qiu BQ, Zhang PF, Xiong D, Xu JJ, Long X, Zhu SQ, Ye XD, Wu Y, Pei X, Zhang XM and Wu YB: CircRNA fibroblast growth factor receptor 3 promotes tumor progression in non-small cell lung cancer by regulating galectin-1-AKT/ERK1/2 signaling. J Cell Physiol 234: 11256-11264, 2019.

43. Zhang S, Liu J, Yuan T, Liu H, Wan C and Le Y: Circular RNA 0001313 knockdown suppresses non-small cell lung cancer cell proliferation and invasion via the microRNA-452/HMGB3/ERK/MAPK axis. Int J Gen Med 13 1495-1507, 2020

44. Bray F, Ferlay J, Soerjomataram I, Siegel RL, Torre LA and Jemal A: Global cancer statistics 2018: GLOBOCAN estimates of incidence and mortality worldwide for 36 cancers in 185 countries. CA Cancer J Clin 68: 394-424, 2018.

45. Hu J, Li H, Wu C, Zhao X and Liu C: The prognostic value of decreased KLF4 in digestive system cancers: A meta-analysis from 17 studies. Dis Markers 2017: 3064246, 2017.

46. Sun C, Li G and Liu M: A novel circular RNA, circ 0005394 , predicts unfavorable prognosis and contributes to hepatocellular carcinoma progression by regulating miR-507/E2F3 and miR-515-5p/CXCL6 signaling pathways. Onco Targets Ther 13: 6171-6180, 2020.

47. Llovet JM and Bruix J: Molecular targeted therapies in hepatocellular carcinoma. Hepatology 48: 1312-1327, 2008.

48. Zhang X, Wang X, Wu T, Li B, Liu T, Wang R, Liu Q, Liu Z, Gong $\mathrm{Y}$ and Shao $\mathrm{C}$ : Isoliensinine induces apoptosis in triple-negative human breast cancer cells through ROS generation and p38 MAPK/JNK activation. Sci Rep 5: 12579, 2015.

49. Zou P, Zhang J, Xia Y, Kanchana K, Guo G, Chen W, Huang Y, Wang Z, Yang S and Liang G: ROS generation mediates the anti-cancer effects of WZ35 via activating JNK and ER stress apoptotic pathways in gastric cancer. Oncotarget 6: 5860-5876, 2015.

50. Xu L, Feng X, Hao X, Wang P, Zhang Y, Zheng X, Li L, Ren S, Zhang $\mathrm{M}$ and Xu M: CircSETD3 (Hsa_circ_0000567) acts as a sponge for microRNA-421 inhibiting hepatocellular carcinoma growth. J Exp Clin Cancer Res 38: 98, 2019.

51. Li W, Zhou X, Wu X, Wei J and Huang Z: The role of circular RNA hsa circ 0085616 in proliferation and migration of hepatocellular carcinoma cells. Cancer Manag Res 11: 7369-7376, 2019.

52. Zhan W, Liao X, Chen Z, Li L, Tian T, Yu L, Wang W and Hu Q Circular RNA hsa circRNA 103809 promoted hepatocellular carcinoma development by regulating miR-377-3p/FGFR1/ERK axis. J Cell Physiol 235: 1733-1745, 2020.

53. Ahmad I, Iwata T and Leung HY: Mechanisms of FGFR-mediated carcinogenesis. Biochim Biophys Acta 1823: 850-860, 2012.

54. Qin M, Liu G, Huo X, Tao X, Sun X, Ge Z, Yang J, Fan J, Liu L and Qin W: Hsa_circ_0001649: A circular RNA and potential novel biomarker for hepatocellular carcinoma. Cancer Biomark 16: 161-169, 2016.

55. Xu Y, Yao Y, Zhong X, Leng K, Qin W, Qu L, Cui Y and Jiang X: Downregulated circular RNA hsa_circ 0001649 regulates proliferation, migration and invasion in cholangiocarcinoma cells. Biochem Biophys Res Commun 496: 455-461, 2018.

56. Li WH, Song YC, Zhang H, Zhou ZJ, Xie X, Zeng QN, Guo K, Wang T, Xia P and Chang DM: Decreased expression of Hsa circ 00001649 in gastric cancer and its clinical significance. Dis Markers 2017: 4587698, 2017.

57. Sun H, Wang Q, Yuan G, Quan J, Dong D, Lun Y and Sun B Hsa_circ_0001649 restrains gastric carcinoma growth and metastasis by downregulation of miR-20a. J Clin Lab Anal 34 e23235, 2020

58. Hsiao YC, Yeh MH, Chen YJ, Liu JF, Tang CH and Huang WC: Lapatinib increases motility of triple-negative breast cancer cells by decreasing miRNA-7 and inducing Raf-1/MAPK-dependent interleukin-6. Oncotarget 6: 37965-37978, 2015. 
59. Weng W, Wei Q, Toden S, Yoshida K, Nagasaka T, Fujiwara T, Cai S, Qin H, Ma Y and Goel A: Circular RNA ciRS-7-A circular RNA ciRS-7-A promising prognostic biomarker and a potential therapeutic target in colorectal cancer. Clin Cancer Res 23 3918-3928, 2017.

60. Pennathur A, Gibson MK, Jobe BA and Luketich JD: Oesophageal carcinoma. Lancet 381: 400-412, 2013.

61. Shi Y, Fang N, Li Y, Guo Z, Jiang W, He Y, Ma Z and Chen Y: Circular RNA LPAR3 sponges microRNA-198 to facilitate esophageal cancer migration, invasion, and metastasis. Cancer Sci 111: 2824-2836, 2020.

62. Gao L, Dou ZC, Ren WH, Li SM, Liang X and Zhi KQ: CircCDR1as upregulates autophagy under hypoxia to promote tumor cell survival via AKT/ERK $\mathrm{E}_{1 / 2} / \mathrm{mTOR}$ signaling pathways in oral squamous cell carcinomas. Cell Death Dis 10: 745, 2019.

63. Deng W, Peng W, Wang T, Chen J, Qiu X, Fu L and Zhu S: Microarray profile of circular RNAs identifies hsa_circRNA_102459 and hsa_circRNA_043621 as important regulators in oral squamous cell carcinoma. Oncol Rep 42: 2738-2749, 2019

64. Li Z, Chen Z, Hu G and Jiang Y: Roles of circular RNA in breast cancer: Present and future. Am J Transl Res 11: 3945-3954, 2019.

65. Gao D, Qi X, Zhang X, Fang K, Guo Z and Li L: hsa circRNA_0006528 as a competing endogenous RNA promotes human breast cancer progression by sponging miR-7-5p and activating the MAPK/ERK signaling pathway. Mol Carcinog 58: 554-564, 2019

66. Wu F and Zhou J: CircAGFG1 promotes cervical cancer progression via miR-370-3p/RAF1 signaling. BMC Cancer 19: 1067, 2019.

67. Huang P, Qi B, Yao H, Zhang L, Li Y and Li Q: Circular RNA cSMARCA5 regulates the progression of cervical cancer by acting as a microRNA-432 sponge. Mol Med Rep 21: 1217-1223, 2020 .

68. Huang XB, Song KJ, Chen GB, Liu R, Jiang ZF and He YL: Circular RNA hsa circ 0003204 promotes cervical cancer cell proliferation, migration, and invasion by regulating MAPK pathway. Cancer Biol Ther 21: 972-982, 2020.

69. van Son M, Peters M, Moerland M, Kerkmeijer L, Lagendijk J and van der Voort van Zyp J: Focal salvage treatment of radiorecurrent prostate Cancer: Anarrative review of current strategies and future perspectives, Cancers (Basel) 10: 480, 2018

70. Yan Z, Xiao Y, Chen Y and Luo G: Screening and identification of epithelial-to-mesenchymal transition-related circRNA and miRNA in prostate cancer. Pathol Res Pract 216: 152784, 2020.

71. Si-Tu J, Cai Y, Feng T, Yang D, Yuan S, Yang X, He S, Li Z, Wang Y, Tang Y, et al: Upregulated circular RNA circ-102004 that promotes cell proliferation in prostate cancer. Int J Biol Macromol 122: 1235-1243, 2019.

72. Lenis AT, Lec PM, Chamie K and Mshs MD: Bladder cancer: A review. JAMA 324: 1980-1991, 2020.

73. Antoni S, Ferlay J, Soerjomataram I, Znaor A, Jemal A and Bray F: Bladder cancer incidence and mortality: A global overview and recent trends. Eur Urol 71: 96-108, 2017.

74. Sun M, Zhao W, Chen Z, Li M, Li S, Wu B and Bu R: Circular RNA CEP128 promotes bladder cancer progression by regulating Mir-145-5p/Myd88 via MAPK signaling pathway. Int J Cancer 145: 2170-2181, 2019.

75. Zhong Z, Huang M, Lv M, He Y, Duan C, Zhang L and Chen J: Circular RNA MYLK as a competing endogenous RNA promotes bladder cancer progression through modulating VEGFA/VEGFR2 signaling pathway. Cancer Lett 403: 305-317, 2017.

76. Lu HC, Yao JQ, Yang X, Han J, Wang JZ, Xu K, Zhou R, $\mathrm{Yu} \mathrm{H}, \mathrm{Lv} \mathrm{Q}$ and $\mathrm{Gu} \mathrm{M}$ : Identification of a potentially functional circRNA-miRNA-mRNA regulatory network for investigating pathogenesis and providing possible biomarkers of bladder cancer. Cancer Cell Int 20: 31, 2020

77. Li J, Huang C, Zou Y, Ye J, Yu J and Gui Y: CircTLK1 promotes the proliferation and metastasis of renal cell carcinoma by sponging miR-136-5p. Mol Cancer 19: 103, 2020.

78. Chen Q, Liu T, Bao Y, Zhao T, Wang J, Wang H, Wang A, Gan X, Wu Z and Wang L: CircRNA cRAPGEF5 inhibits the growth and metastasis of renal cell carcinoma via the miR-27a-3p/TXNIP pathway. Cancer Lett 469: 68-77, 2020.

79. Liu H, Bi J, Dong W, Yang M, Shi J, Jiang N, Lin T and Huang J: Invasion-related circular RNA circFNDC3B inhibits bladder cancer progression through the miR-1178-3p/G3BP2/SRC/FAK axis. Mole-cular Cancer 17: 161, 2018.

80. Chen T, Yu Q, Shao S and Guo L: Circular RNA circFNDC3B protects renal carcinoma by miR-99a downregulation. J Cell Physiol 235: 4399-4406, 2020.
81. Liu X, Zhou C, Li Y, Deng Y, Lu W and Li J: Upregulation of circ-0000745 in acute lymphoblastic leukemia enhanced cell proliferation by activating ERK pathway. Gene 751: 144726, 2020.

82.Zhou F, Wang D, Wei W, Chen H, Shi H, Zhou N, Wu L and Peng R: Comprehensive profiling of circular RNA expressions reveals potential diagnostic and prognostic biomarkers in multiple myeloma. BMC Cancer 20: 40, 2020.

83. Zheng J, Liu X, Xue Y, Gong W, Ma J, Xi Z, Que Z and Liu Y: TTBK 2 circular RNA promotes glioma malignancy by regulating miR-217/HNF1ß/Derlin-1 pathway. J Hematol Oncol 10: 52, 2017.

84. Gerecke C, Fuhrmann S, Strifler S, Schmidt-Hieber M, Einsele $\mathrm{H}$ and Knop S: The diagnosis and treatment of multiple myeloma. Dtsch Arztebl Int 113: 470-476, 2016.

85. Brigle K and Rogers B: Pathobiology and diagnosis of multiple myeloma. Semin Oncol Nurs 33: 225-236, 2017.

86. Joshua DE, Bryant C, Dix C, Gibson J and Ho J: Biology and therapy of multiple myeloma. Med J Aust 210: 375-380, 2019.

87. Ring ES, Lawson MA, Snowden JA, Jolley I and Chantry AD: New agents in the treatment of myeloma bone disease. Calcif Tissue Int 102: 196-209, 2018.

88. Kim H, Zheng S, Amini SS, Virk SM, Mikkelsen T, Brat DJ, Grimsby J, Sougnez C, Muller F, Hu J, et al: Whole-genome and multisector exome sequencing of primary and post-treatment glioblastoma reveals patterns of tumor evolution. Genome Res 25: 316-327, 2015.

89. He J, Huang Z, He M, Liao J, Zhang Q, Wang S, Xie L, Ouyang L, Koeffler HP, Yin D and Liu A: Circular RNA MAPK4 (circ-MAPK4) inhibits cell apoptosis via MAPK signaling pathway by sponging miR-125a-3p in gliomas. Mol Cancer 19: 17, 2020.

90. Ko B, He T, Gadgeel S and Halmos B: MET/HGF pathway activation as a paradigm of resistance to targeted therapies. Ann Transl Med 5: 4, 2017.

91. Pozzi C, Cuomo A, Spadoni I, Magni E, Silvola A, Conte A, Sigismund S, Ravenda PS, Bonaldi T, Zampino MG, et al: The EGFR-specific antibody cetuximab combined with chemotherapy triggers immunogenic cell death. Nat Med 22: 624-631, 2016.

92. Jeyaraman S, Hanif EAM, Ab Mutalib NS, Jamal R and Abu N: Circular RNAs: Potential regulators of treatment resistance in human cancers. Front Genet 10: 1369, 2020

93. Neuzillet C, Tijeras-Raballand A, de Mestier L, Cros J, Faivre S and Raymond E: MEK in cancer and cancer therapy. Pharmacol Ther 141: 160-171, 2014.

94. Li M, Meng F and Lu Q: expression profile screening and bioinformatics analysis of circRNA, LncRNA, and mRNA in acute myeloid leukemia drug-resistant cells. Turk J Haematol 37: 104-110, 2020

95. Salzman J, Chen RE, Olsen MN, Wang PL and Brown PO Cell-type specific features of circular RNA expression. PLoS Genet 9: e1003777, 2013

96. Böhmdorfer G and Wierzbicki AT: Control of chromatin structure by long noncoding RNA. Trends Cell Biol 25: 623-632, 2015

97. Du WW, Yang W, Liu E, Yang Z, Dhaliwal P and Yang BB: Foxo3 circular RNA retards cell cycle progression via forming ternary complexes with p21 and CDK2. Nucleic Acids Res 44: 2846-2858, 2016

98. Holdt LM, Stahringer A, Sass K, Pichler G, Kulak NA, Wilfert W, Kohlmaier A, Herbst A, Northoff BH, Nicolaou A, et al: Circular non-coding RNA ANRIL modulates ribosomal RNA maturation and atherosclerosis in humans. Nat Commun 7: 12429, 2016

99. Yaeger R and Corcoran RB: Targeting alterations in the RAF-MEK pathway. Cancer Discov 9: 329-341, 2019.

100. McCubrey JA, Rakus D, Gizak A, Steelman LS, Abrams SL, Lertpiriyapong K, Fitzgerald TL, Yang LV, Montalto G,

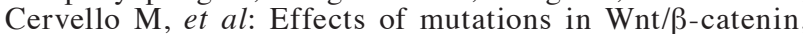
hedgehog, notch and PI3K pathways on GSK-3 activity-diverse effects on cell growth, metabolism and cancer. Biochim Biophys Acta 1863: 2942-2976, 2016.

101. Wu C, Zhuang Y, Jiang S, Liu S, Zhou J, Wu J, Teng Y, Xia B, Wang $R$ and Zou $X$ : Interaction between Wnt/ $\beta$-catenin pathway and microRNAs regulates epithelial-mesenchymal transition in gastric cancer (Review). Int J Oncol 48: 2236-2246, 2016.

102. Guo YH, Wang LQ, Li B, Xu H, Yang JH, Zheng LS, Yu P, Zhou AD, Zhang Y, Xie SJ, et al: Wnt/ $\beta$-catenin pathway transactivates microRNA-150 that promotes EMT of colorectal cancer cells by suppressing CREB signaling. Oncotarget 7: 42513-42526, 2016.

This work is licensed under a Creative Commons Attribution-NonCommercial-NoDerivatives 4.0 International (CC BY-NC-ND 4.0) License. 